\title{
AN EXTREMAL PROBLEM FOR FUNCTIONS OF POSITIVE REAL PART WITH APPLICATION TO A RADIUS OF CONVEXITY PROBLEM
}

\author{
D. H. HAMILTON ${ }^{1}$ AND P. D. TUAN
}

\begin{abstract}
The functional $\operatorname{Re}\left\{z p^{\prime}(z) /(p(z)+\beta+i t)\right\}, \beta>-1,|z|<r$, $0<r<1$, is minimized for all real $t$ over the class of functions of positive real part. The result is applied to obtain the radius of convexity for a family of regular functions.
\end{abstract}

1. Introduction. Let $S^{*}(\sigma), \sigma<1$, be the class of functions $g(z)$ regular in $\Delta=\{z:|z|<1\}$ such that $g(0)=0, g^{\prime}(0)=1$ and $\operatorname{Re}\left\{z g^{\prime}(z) / g(z)\right\}>\sigma$ for all $z \in \Delta$.

We define the class $K(\sigma, \lambda), \sigma<1, \lambda<1$, to be the set of functions $f(z)$ with $f(0)=0, f^{\prime}(0)=1$, for which there exist $g(z) \in S^{*}(\sigma)$ and a real number $\alpha,|\alpha|<\pi / 2$, such that

$$
\operatorname{Re}\left\{e^{i \alpha}\left[z f^{\prime}(z) / g(z)-\lambda\right]\right\}>0 \text { for } z \in \Delta .
$$

The radius of convexity of $K(\sigma, \lambda)$ is the greatest value of $r, 0<r \leqslant 1$, for which $\operatorname{Re}\left\{1+z f^{\prime \prime}(z) / f(z)\right\}>0$ for $|z|<r$ and for all $f(z)$ in $K(\sigma, \lambda)$. Jablonski and Wesolowski [1] found a lower bound for the radius of convexity of $K(0, \lambda)$ but the result is not sharp. Jankovics [2] obtained the radius of starlikeness of the class $\left\{f(z): \operatorname{Re}\left\{e^{i \alpha}[f(z) / z-\lambda]\right\}>0, z \in \Delta\right\}$ by means of some results of Ruscheweyh [6]. The latter problem turns out to be equivalent to the former one with $g(z) \equiv z$.

We approach the problem of finding the radius of convexity of $K(\sigma, \lambda)$ by first determining

$$
M(\beta, r)=\min _{t \in \mathbf{R}}\{M(\beta, r, t)\}
$$

where

$$
M(\beta, r, t)=\min _{\substack{p \in P \\|z|<r}}\left\{\operatorname{Re} \frac{z p^{\prime}(z)}{p(z)+\beta+i t}\right\},
$$

for $0 \leqslant r<1, \beta>0$ and $P$ denoting the class of regular functions $p(z)=1$ $+\sum_{k=1}^{\infty} c_{k} z^{k}$ and $\operatorname{Re}\{p(z)\}>0$ for $z \in \Delta$. This extremal problem is well known and interesting on its own. Robertson [5], by means of a variational

Received by the editors July 11, 1977 and, in revised form, February 20, 1978.

AMS (MOS) subject classifications (1970). Primary 30A40, 30A32.

Key words and phrases. Extremal problem, radius of convexity, functions of positive real part.

'This author acknowledges the financial support of a University of Tasmania Research Studentship.

๑ American Mathematical Society 1978 
method, showed that the extremal functions in (1) have the form

$$
p_{0}(z)=\gamma \frac{1+z e^{i \theta_{1}}}{1-z e^{i \theta_{1}}}+(1-\gamma) \frac{1+z e^{i \theta_{2}}}{1-z e^{i \theta_{2}}}
$$

where $0 \leqslant \gamma \leqslant 1,0 \leqslant \theta_{1} \leqslant 2 \pi, 0 \leqslant \theta_{2} \leqslant 2 \pi$ and the parameters $\gamma, \theta_{1}, \theta_{2}$ are to be determined. Zmorovič [8] investigated $M(\beta, r, t)$ using Robertson's result but was only able to determine $M(0, r, t)$ and $M(\beta, r, 0)$ while Robertson [5] has previously found $M(0, r)$. We obtain $M(\beta, r)$ by minimizing the functional first with respect to $t$ and then over the class $P$.

2. The extremal problem. Before stating the main result of this section we need three lemmas. Using bilinear maps we may easily prove

LEMMA 1. Let $a$ and $b$ be complex numbers such that $\operatorname{Re} b>0$ then

$$
\operatorname{Re}\left\{\frac{a}{b+i t}\right\} \geqslant \frac{\operatorname{Re}\{a\}-|a|}{2 \operatorname{Re}\{b\}} \quad(t \in \mathbf{R}) .
$$

Next, let $H$ be the set of functions $f(z)$ regular in $\Delta$. A real functional $\phi$ defined on a convex subset $F$ of $H$ is said to be convex on $F$ if

$$
\phi(\gamma x+(1-\gamma) y) \leqslant \gamma \phi(x)+(1-\gamma) \phi(y)
$$

for any $\gamma, 0 \leqslant \gamma \leqslant 1$, and $x, y$ in $F$. If equality always holds then $\phi$ is said to be affine on $F$.

A function $f_{0} \in F$ is said to be an extreme point of $F$ if we cannot write $f_{0}=\gamma f_{1}+(1-\gamma) f_{2}$, for some $\gamma, 0<\gamma<1$, and distinct functions $f_{1}, f_{2}$ in $F$. Let $E_{F}$ denote the set of extreme points of $F$. Then we have

LEMMA 2. [4]. Let - $\phi$ be a convex real functional on a compact convex subset $F$ of $H$. Then there always exists $f_{1} \in E_{F}$ such that $\min _{f \in F} \phi(f)=\phi\left(f_{1}\right)$.

The next result is a generalization of a theorem by Ruscheweyh [7].

Lemma 3. Let $F$ be a compact convex set in $H$. Suppose that the real functionals $-\phi$ and $\psi$ are convex and affine on $F$ respectively. If $\psi(f)>0$ for all $f \in F$, then $\min _{F}\{\phi(f) / \psi(f)\}=\left\{\phi\left(f_{1}\right) / \psi\left(f_{1}\right)\right\}$, for some $f_{1} \in E_{F}$.

Proof. Define $d=\min _{F}\{\phi(f) / \psi(f)\}=\phi\left(f_{0}\right) / \psi\left(f_{0}\right)$, some $f_{0} \in F$. Clearly $0 \leqslant \phi(f)-d \psi(f)$ and thus $0=\min _{F}\{\phi(f)-d \psi(f)\}$. But $d \psi(f)-\phi(f)$ is convex and hence by Lemma 2 , there exists $f_{1} \in E_{F}$ such that $0=\phi\left(f_{1}\right)$ $d \psi\left(f_{1}\right)$, and so $d=\phi\left(f_{1}\right) / \psi\left(f_{1}\right)$.

TheOREM 1. For $\beta>0, p \in P, t \in \mathbf{R},|z| \leqslant r$,

(a) $\operatorname{Re}\left\{\frac{z p^{\prime}(z)}{p(z)+\beta+i t}\right\} \geqslant \frac{-2 r}{(1+r)[\beta(1+r)+(1-r)]}$, for $r \leqslant r_{0}$,

(b) $\operatorname{Re}\left\{\frac{z p^{\prime}(z)}{p(z)+\beta+i t}\right\} \geqslant \frac{(1+r)^{2}\left[(1-r)^{2}-u_{1}\right]}{2 u_{1}\left(1-r^{2}+\beta u_{1}\right)}$, for $r \geqslant r_{0}$, 
where $u_{1}=(1-r)^{2}\left[1+\{1+(1+r) / \beta(1-r)\}^{1 / 2}\right]$, and $r_{0}=\left(x_{0}-1\right) /\left(x_{0}\right.$ $+1), x_{0}$ being the positive root of the equation $\beta x^{3}-2 \beta x-1=0$.

For $-1<\beta \leqslant 0, p \in P, t \in \mathbf{R},|z| \leqslant r<(1+\beta) /(1-\beta)$, (a) also holds.

Proof. If $\operatorname{Re}\{p(z)+\beta\}>0$ then from Lemma 1

$$
\min _{t \in \mathbf{R}} \operatorname{Re}\left\{\frac{z p^{\prime}(z)}{p(z)+\beta+i t}\right\}=\frac{\operatorname{Re}\left\{z p^{\prime}(z)\right\}-\left|z p^{\prime}(z)\right|}{2 \operatorname{Re}\{p(z)+\beta\}} .
$$

It can be verified that $-\phi(p) \equiv\left|z p^{\prime}(z)\right|-\operatorname{Re}\left\{z p^{\prime}(z)\right\}$ is convex on $P$ and that $\psi(p) \equiv 2 \operatorname{Re}\{p(z)+\beta\}$ is affine on $P$. Also, $\psi(p)>0$ for all $p \in P$, $|z| \leqslant r<1$ if $\beta \geqslant 0$, and $\psi(p)>0$ for all $p \in P,|z| \leqslant r<(1+\beta) /(1-\beta)$ if $-1<\beta \leqslant 0$. Since $P$ is compact and convex, we may apply Lemma 3 to obtain

$$
\min _{p \in P} \frac{\operatorname{Re}\left\{z p^{\prime}(z)\right\}-\left|z p^{\prime}(z)\right|}{2 \operatorname{Re}\{p(z)+\beta\}}=\frac{\operatorname{Re}\left\{z p_{1}^{\prime}(z)\right\}-\left|z p_{1}^{\prime}(z)\right|}{2 \operatorname{Re}\left\{p_{1}(z)+\beta\right\}},
$$

for some $p_{1}(z) \in E_{p}$. But

$$
E_{p}=\left\{\frac{1+z e^{i \theta}}{1-z e^{i \theta}}: 0 \leqslant \theta \leqslant 2 \pi\right\} .
$$

Thus,

$$
M(\beta, r)=\min _{0<\theta<2 \pi} \frac{r(1+r)^{2}(\cos \theta-1)}{\left[\beta\left(1-2 r \cos \theta+r^{2}\right)+1-r^{2}\right]\left[1-2 r \cos \theta+r^{2}\right]} .
$$

On making the substitution $u=1-2 r \cos \theta+r^{2}$, we can write

$$
M(\beta, r)=\min _{m<u<n} s(u),
$$

where $m=(1-r)^{2}, n=(1+r)^{2}$ and

$$
s(u)=n(m-u) / 2 u\left(1-r^{2}+\beta u\right) .
$$

Now

$$
s^{\prime}(u)=\frac{n}{2} \cdot \frac{\beta u^{2}-2 m \beta u-m\left(1-r^{2}\right)}{u^{2}\left(1+\beta u-r^{2}\right)^{2}} .
$$

For $-1<\beta \leqslant 0$ and $r<(1+\beta) /(1-\beta), s^{\prime}(u)<0$, therefore in this case we find

$$
M(\beta, r)=s(n)=-\frac{2 r}{(1+r)[\beta(1+r)+(1-r)]} .
$$

For $\beta>0, s^{\prime}(u)$ has zeros at

$$
\begin{aligned}
& u_{1} \\
& u_{2}
\end{aligned} \equiv m \pm\left[m^{2}+m\left(1-r^{2}\right) / \beta\right]^{1 / 2}
$$

Since $u_{2}<0<m$ and $u_{1}>m, M(\beta, r)=s\left(u_{1}\right)$ if $u_{1} \leqslant n$, otherwise $M(\beta, r)$ 
$=s(n)$. The condition $u_{1} \geqslant n$ is equivalent to

$$
(1-r)^{2}+\left[(1-r)^{4}+(1-r)^{2}\left(1-r^{2}\right) / \beta\right]^{1 / 2} \geqslant(1+r)^{2}
$$

which, on putting $x=(1+r) /(1-r) \geqslant 1$, becomes

$$
1+(1+x / \beta)^{1 / 2} \geqslant x^{2} \text {. }
$$

Thus $(1+x / \beta)^{1 / 2} \geqslant x^{2}-1 \geqslant 0$ and consequently $u_{1} \geqslant n$ if $f(x)=\beta x^{3}-$ $2 \beta x-1 \geqslant 0$. Our result follows from the fact that $f(x)$ has exactly one positive root, say $x_{0}$, in $[1, \infty)$.

3. Radius of convexity of $K(\sigma, \lambda)$.

THEOREM 2. The radius of convexity $r_{c}$ of $K(\sigma, \lambda)$ is given by

(a) the least positive root of

$$
\begin{aligned}
0=\sigma+(1-\sigma) \frac{1-r}{1+r}-\frac{2 r}{(1+r)[\beta(1+r)+(1-r)]} \\
\quad(\beta=\lambda /(1-\lambda))
\end{aligned}
$$

when $\lambda \leqslant \lambda_{0}=\left(x^{3}-2 x+1\right)^{-1}$, where $x=\sigma+\left(\sigma^{2}-2 \sigma+4\right)^{1 / 2}$,

(b) the least positive root of

$$
0=\sigma+(1-\sigma) \frac{1-r}{1+r}+\frac{(1+r)^{2}}{2} \frac{\left(1-r^{2}-u_{1}\right)}{u_{1}\left(1-r^{2}+\beta u_{1}\right)}
$$

when $\lambda \geqslant \lambda_{0}$, where $u_{1}=(1-r)^{2}\left[1+(1+(1+r) / \beta(1-r))^{1 / 2}\right]$. These results are sharp.

Proof. We have that $f(z) \in K(\sigma, \lambda)$ if and only if $\left[z f^{\prime}(z) / g(z)-\lambda\right] /(1-$ $\lambda$ ) is subordinate to

$$
\frac{1+c z}{1-z}=\frac{1}{2}(1-c)+\frac{1}{2}(1+c) \frac{1+z}{1-z}
$$

where $c=e^{-2 i \alpha}$. Hence, there exists $p(z) \in P$ such that

$$
f^{\prime}(z)=z^{-1} g(z)\left\{\lambda+(1-\lambda)\left[\frac{1}{2}(1-c)+\frac{1}{2}(1+c) p(z)\right]\right\} .
$$

We thus find

$$
1+\frac{z f^{\prime \prime}(z)}{f^{\prime}(z)}=\frac{z g^{\prime}(z)}{g(z)}+\frac{z p^{\prime}(z)}{(1-c) /(1+c)(1-\lambda)+\lambda /(1-\lambda)+p(z)} \text {. }
$$

Now as $c=e^{-2 i \alpha}$

$$
\frac{\lambda}{1-\lambda}+\frac{1-c}{1+c} \frac{1}{1-\lambda}=\beta+i t
$$

where $\beta=\lambda /(1-\lambda)$ and $t=(\tan \alpha) /(1-\lambda)$. It is clear that all possible values of $t$ are taken as $\alpha$ varies in $\left(-\frac{1}{2} \pi, \frac{1}{2} \pi\right)$. Thus

$$
\operatorname{Re}\left\{1+\frac{z f^{\prime \prime}(z)}{f^{\prime}(z)}\right\}=\operatorname{Re}\left\{\frac{z g^{\prime}(z)}{g(z)}\right\}+\operatorname{Re}\left\{\frac{z p^{\prime}(z)}{p(z)+\beta+i t}\right\} .
$$


For $g(z) \in S^{*}(\sigma)$, it is well known that

$$
\operatorname{Re}\left\{\frac{z g^{\prime}(z)}{g(z)}\right\} \geqslant \sigma+(1-\sigma) \frac{1-r}{1+r} .
$$

From (5), (6) and Theorem 1 we obtain the required result. It remains to determine $\lambda_{0}$. When $\lambda=\lambda_{0}$, we must have that the radii of convexity in the two cases coincide, say $r_{c}=R$. Then, according to Theorem 1 and part (a) of this theorem, the values of $R$ and $\beta_{0}=\lambda_{0} /\left(1-\lambda_{0}\right)$ are determined by

$$
\beta_{0} x^{3}-2 \beta_{0} x-1=0, \quad x=(1+R) /(1-R)>1,
$$

and

$$
0=\sigma+(1-\sigma) \frac{1-R}{1+R}-\frac{2 R}{(1+R)\left[\beta_{0}(1+R)+(1-R)\right]}
$$

Elimination of $\beta_{0}$ and $R$ from these equations gives

$$
\left(x^{2}-2 \sigma x+2 \sigma-4\right)=0 .
$$

It follows that $x=\sigma+\left(\sigma^{2}-2 \sigma+4\right)^{1 / 2}$ and hence $\lambda_{0}$ is (uniquely) determined and the proof of Theorem 2 is completed.

REMARK 1. For $\lambda \leqslant \lambda_{0}$, the extremal function is

$$
f_{0}(z)=\int_{0}^{z}\left\{\lambda+(1-\lambda) \frac{1+\xi}{1-\xi}\right\} \frac{d \xi}{(1-\xi)^{2(1-\sigma)}} .
$$

For $\lambda \geqslant \lambda_{0}$, the extremal functions are

$$
f_{0}(z)=\int_{0}^{z}\left\{\lambda+\frac{1-\lambda}{2}\left[\left(1-c_{0}\right)+\left(1+c_{0}\right) \frac{1+e^{i \theta_{0} \xi}}{1-e^{i \theta_{0} \xi}}\right]\right\} \frac{d \xi}{(1-\xi)^{2(1-\sigma)}}
$$

where $\cos \theta_{0}=\left(1+r^{2}-u_{1}\right) / 2 r$ and $c_{0}=\left[(1-\lambda) i t_{0}-1\right] /\left[(1-\lambda) i t_{0}+1\right]$ with $u_{1}$ as defined in Theorem 1 and

$$
t_{0}=\frac{2 r \sin \theta_{0}}{u_{1}}\left\{\frac{\left(1-r^{2}+\beta u_{1}\right)(1-r)}{(1+r)^{2}\left[(1-r)^{2}-u_{1}\right]}-1\right\}
$$

Putting $g(z) \equiv z$, we recover Jankovics' result [2]. For $\sigma=0$, our result differs from that of Jablonski and Wesolowski [1] as expected.

REMARK 2. Libera [3] considered the class $C(\sigma, \lambda)$ of functions $f(z)$ such that $\operatorname{Re}\left\{e^{i \alpha} z f^{\prime}(z) / g(z)\right\}>\lambda$ for some $g(z) \in S^{*}(\sigma)$. Libera was only able to place a lower bound on the radius of convexity of $C(\sigma, \lambda)$. Now, since $C(\sigma, \lambda) \subseteq K(\sigma, \lambda), r_{c}$ for $K(\sigma, \lambda)$ is another lower bound for the radius of convexity of $C(\sigma, \lambda)$. However, for the case $\lambda \leqslant \lambda_{0}$, as the extremal function $f_{0}(z)$ belongs to $C(\sigma, \lambda)$, we conclude that $r_{c}$ is also the radius of convexity for $C(\sigma, \lambda)$. 


\section{REFERENCES}

1. F. Jablonski and A. Weslowski, Sur une famille de fonctions holomorphes dans le cercle unité, Ann. Univ. Mariae Curie-Skłodowska Sect. A 21 (1967), 91-99.

2. R. Jankovics, Uber Funktionen mit der Eigenschaft $\operatorname{Re}\left\{e^{i \alpha}(f(z) / z-\beta)\right\}>0$, Math. Z. 143 (1975), 235-242.

3. R. Libera, Some radius of convexity problems, Duke Math. J. 31 (1964), 143-158.

4. A. W. Roberts and D. A. Varberg, Convex functions, Academic Press, New York, 1973.

5. M. S. Robertson, Extremal problems for analytic functions with positive real part and applications, Trans. Amer. Math. Soc. 106 (1963), 236-253.

6. S. Ruscheweyh, Duality for Hadamard products with applications to extremal problems for functions regular in the unit disk, Trans. Amer. Math. Soc. 210 (1975), 63-74.

7. $\quad$ Nichtlineare extremal probleme für holomorphe Stieltjesintegrale, Math. Z. 142 (1975), 19-23.

8. V. A. Zmorovic, On the bounds of starlikeness and of univalence in certain classes of functions regular in the circle $|z|<1$, Ukrain. Mat. Z. 18 (1966), 28-39.

Department of Mathematics, University of Tasmania, Hobart, Tasmania, Australia 